Fetal Diagnosis aud Therapy
Fetal Diagn Ther 2014;35:240-248

DOI: $10.1159 / 000359968$
Received: November 25, 2013

Accepted after revision: January 12, 2014

Published online: May 17, 2014

\title{
Competing Risks Model in Screening for Preeclampsia by Serum Placental Growth Factor and Soluble fms-Like Tyrosine Kinase-1 at 30-33 Weeks' Gestation
}

\author{
Jonathan Lai ${ }^{\mathrm{a}}$ Santiago Garcia-Tizon Larroca ${ }^{\mathrm{a}}$ Gergana Peeva ${ }^{\mathrm{a}}$ Leona C. Poon ${ }^{\mathrm{a}}$ \\ David Wright $^{\text {b }}$ Kypros H. Nicolaides ${ }^{\text {a }}$ \\ ${ }^{a}$ Harris Birthright Research Centre of Fetal Medicine, King's College Hospital, London, and b Institute of Health Research, \\ University of Exeter Medical School, Exeter, UK
}

\section{Key Words}

Third-trimester screening · Preeclampsia - Placental growth factor . Soluble fms-like tyrosine kinase- 1 - Pyramid of antenatal care

\begin{abstract}
Objective: To assess the risk for preeclampsia (PE) by maternal characteristics, serum placental growth factor (PIGF) and soluble fms-like tyrosine kinase-1 (sFlt-1) at 30-33 weeks' gestation. Methods: This was a screening study in singleton pregnancies including 2,140 that subsequently developed $\mathrm{PE}$ and 83,615 that were unaffected by $\mathrm{PE}$, gestational hypertension or delivery of small-for-gestational-age neonates (normal group). We developed a survival time model for the time of delivery for PE by combination of maternal characteristics and history with PIGF and sFlt-1 multiple of the median (MoM) values (biochemical test). Data on third-trimester PIGF and sFlt- 1 were available in 118 cases of PE and 3,734 of normal group. The detection rate (DR) of PE requiring delivery within 4, 6 and 8 weeks of the visit was estimated. $R \boldsymbol{e}$ sults: In pregnancies with PE, the $\log _{10}$ MoM values of PIGF and sFlt-1 were linearly related to gestational age at delivery. Screening by the biochemical test detected 100,76 , and $62 \%$ of PE with delivery within 4, 6 and 8 weeks of the visit, at a fixed false-positive rate of $5 \%$. Interpretation: Testing by
\end{abstract}

PIGF and sFIt-1 at 30-33 weeks could identify all pregnancies developing $\mathrm{PE}$ and requiring delivery within the subsequent 4 weeks.

(c) 2014 S. Karger AG, Basel

\section{Introduction}

Preeclampsia (PE), which affects $2-3 \%$ of pregnancies and is a major cause of maternal and perinatal morbidity and mortality [1-3], is thought to be the consequence of an imbalance in angiogenic and anti-angiogenic proteins [4]. Several studies have reported that maternal serum levels of placental growth factor (PlGF) are reduced and those of soluble fms-like tyrosine kinase-1 (sFlt-1) are increased in women with PE. There is also evidence that the level of these proteins is altered before the onset of the clinical signs of the disease. However, a meta-analysis of such studies concluded that the test accuracies of serum PlGF and sFlt-1 before 30 weeks' gestation are too poor for accurate prediction of PE in clinical practice [5].

Recent studies have focused on the investigation of pregnancies presenting to specialist clinics with signs of hypertensive disorders with the aim of identifying the subgroup that will develop severe $\mathrm{PE}$ requiring delivery within the subsequent 1-4 weeks. In such high-risk pregnancies,

\section{KARGER}

E-Mail karger@karger.com

www.karger.com/fdt
C 2014 S. Karger AG, Basel

$1015-3837 / 14 / 0354-0240 \$ 39.50 / 0$
Prof. K.H. Nicolaides

Harris Birthright Research Centre for Fetal Medicine

King's College Hospital, Denmark Hill

London SE5 9RS (UK)

E-Mail kypros@fetalmedicine.com 
measurement of serum PlGF or the sFlt- 1 to PlGF ratio are highly accurate in identifying the target group [6-11].

The objective of this screening study is to investigate the potential value of maternal serum concentrations of PlGF, sFlt-1 and their combination as part of routine clinical care at 30-33 weeks' gestation in the prediction of subsequent development of $\mathrm{PE}$.

\section{Methods}

\section{Study Population}

The data for this study were derived from prospective screening for adverse obstetric outcomes in women with singleton pregnancies attending for their routine first- and third-trimester hospital visit at King's College Hospital London and Medway Maritime Hospital Kent between March 2006 and June 2013. The first-trimester visit, at $11^{+0}-13^{+6}$ weeks' gestation, included recording of maternal characteristics and medical history, measurement of maternal weight and height and ultrasound examination for fetal anatomy, screening for aneuploidies and measurement of fetal crown-rump length for assessment of gestational age [12]. The third-trimester visit, at $30^{+0}-33^{+6}$ weeks' gestation, included ultrasound examination for assessment of fetal growth and wellbeing. Maternal blood was collected for research and serum PlGF and sFlt-1 were measured within 15 min of blood sampling (Cobas e411, Roche Diagnostics, Penzberg, Germany). Written informed consent was obtained from the women agreeing to participate in the study, which was approved by the NHS National Research Ethics Service.

Patients were asked to complete a questionnaire on maternal age, racial origin (Caucasian, Afro-Caribbean, South Asian, East Asian and mixed), method of conception (spontaneous or assisted conception requiring the use of ovulation drugs or in vitro fertilisation, IVF), cigarette smoking during pregnancy (yes or no), history of chronic hypertension (yes or no), history of type 1 or 2 diabetes mellitus (yes or no), history of systemic lupus erythematosus (SLE) or antiphospholipid syndrome (APS; yes or no), family history of PE in the mother of the patient (yes or no) and obstetric history including parity (parous or nulliparous if no previous pregnancies at or after 24 weeks), previous pregnancy with PE (yes or no), previous pregnancy with small-for-gestational-age (SGA) babies (yes or no) and inter-pregnancy interval. The questionnaire was then reviewed by a doctor together with the patient. The maternal weight and height were recorded.

\section{Sample Analyses}

Serum PlGF and sFlt-1 were measured in parallel, using an automated electrochemiluminescence immunoassay system (Cobas e411, Roche Diagnostics, Penzberg, Germany). The inter-assay coefficients of variation for the low and high concentrations were 5.4 and $3.0 \%$ for PlGF, and 3.0 and 3.2\% for sFlt-1, respectively. The cobas e411 analyzer PlGF and sFlt-1 assay covers a measurement range from 3 to $10,000 \mathrm{pg} / \mathrm{ml}$ and from 10 to $85,000 \mathrm{pg} / \mathrm{ml}$, respectively.

\section{Outcome Measures}

Data on pregnancy outcome were collected from the hospital maternity records or the general medical practitioners of the wom- en. The obstetric records of all women with pre-existing or pregnancy-associated hypertension were examined to determine if the condition was chronic hypertension, $\mathrm{PE}$ or non-proteinuric gestational hypertension $(\mathrm{GH})$.

The definition of PE was that of the International Society for the Study of Hypertension in Pregnancy [13]. The definition of SGA was birthweight below the 5 th percentile of reference range derived from our population [14].

\section{Statistical Analysis}

Comparisons of maternal characteristics between outcome groups were by $\chi^{2}$ or Fisher's exact test for categorical variables and by Student's t test or Mann-Whitney U test for continuous variables.

Measurements of serum PlGF and sFlt-1 were $\log _{10}$ transformed to produce distributions of residuals approximately Gaussian in shape. Backward stepwise multiple regression analysis was used to determine which of the factors amongst the maternal characteristics and gestation were significant predictors of the $\log _{10}$ PlGF and $\log _{10}$ sFlt-1, adjusting for the adverse pregnancy outcomes as specified (PE, GH and SGA). Variables were excluded from the model if the $p$ value was $>0.05$ or if their effect size was less than one tenth of the $\log _{10}$ multiple of the median (MoM) standard deviation. Maternal age was centred by subtracting 30 years, maternal weight was centred by subtracting $69 \mathrm{~kg}$ and maternal height was centred by subtracting $164 \mathrm{~cm}$. The distribution of PlGF and sFlt- 1 was then expressed as MoM in all cases, correcting for the significant predictors as defined in the multiple regression.

A competing risk model was used to combine the prior information from maternal characteristics with PlGF and sFlt-1 MoM values [15-18]. The distribution of gestational age at delivery with PE was defined by two components: firstly, the prior distribution based on maternal characteristics [17] and secondly, the distribution of PlGF and sFlt-1 MoM values with gestational age in pregnancies affected by PE. In the cases of PE, regression analysis was used to determine the relationship between $\log _{10}$ MoM values with gestational age at delivery.

The model for calculation of the a priori risk, based on maternal characteristics and history, was derived from the study of 2,140 cases of PE and 83,615 unaffected pregnancies and was reported previously [17]. Certain variables, including advancing maternal age over 35 years, increasing weight, Afro-Caribbean and South Asian racial origin, personal or family history of PE, conception by IVF and a medical history of chronic hypertension, diabetes mellitus and SLE or APS increase the risk for development of PE [17]. The consequence of this increased risk is a shift to the left of the gaussian distribution of the gestational age at delivery with PE.

Risks for all $\mathrm{PE}$ and $\mathrm{PE}$ requiring delivery within the subsequent 4,6 and 8 weeks in screening by maternal characteristics, PlGF and sFlt-1, and their combination were computed according to the competing risks model. Detection rates (DRs) at fixed falsepositive rates (FPR) of 5 and $10 \%$ were estimated using these risks.

To provide model-based estimates of screening performance for pregnancies delivering with PE within a specific time of the third trimester assessment, the following procedure was adopted. Firstly, N pregnancy records were produced by sampling with replacement from the data set for which delivery with PE occurred within the specific time window of the third trimester visit. This provided a sample of pregnancies with characteristics representative of the pregnancies in the original data delivering within the specified time window. Secondly, for each of the $\mathrm{N}$ records, the 
Table 1. Characteristics in the study population

\begin{tabular}{|c|c|c|c|c|}
\hline \multirow[t]{2}{*}{ Characteristic } & \multicolumn{2}{|c|}{ Maternal characteristics at $11-13$ weeks } & \multicolumn{2}{|c|}{ PlGF and sFlt- 1 at $30-33$ weeks } \\
\hline & $\begin{array}{l}\text { normal } \\
(\mathrm{n}=83,615)\end{array}$ & $\begin{array}{l}\mathrm{PE} \\
(\mathrm{n}=2,140)\end{array}$ & $\begin{array}{l}\text { normal } \\
(\mathrm{n}=3,734)\end{array}$ & $\begin{array}{l}\mathrm{PE} \\
(\mathrm{n}=118)\end{array}$ \\
\hline Maternal age, years & $31.2(26.8-35.0)$ & $31.2(26.5-35.8)$ & $31.0(26.7-34.6)$ & $31.4(27.0-34.1)$ \\
\hline Maternal weight, kg & $65.7(59.0-75.6)$ & $72.0(62.0-85.3)^{*}$ & $77.0(69.0-88.0)$ & $84.3(74.4-98.1)^{*}$ \\
\hline Maternal height, $\mathrm{cm}$ & $164(160-169)$ & $163(159-167)^{*}$ & $165(160-169)$ & $164(159-168)^{*}$ \\
\hline Caucasian & $63,457(75.9)$ & $1,252(58.5)^{*}$ & $2,892(77.5)$ & $78(66.1)^{*}$ \\
\hline Afro-Caribbean & $11,993(14.3)$ & $684(32.0)^{*}$ & $552(14.8)$ & $32(27.1)^{*}$ \\
\hline South Asian & $4,046(4.8)$ & $119(5.6)$ & $138(3.7)$ & $5(4.2)$ \\
\hline East Asian & $2,125(2.5)$ & $39(1.8)^{*}$ & $69(1.8)$ & $2(1.7)$ \\
\hline Mixed & $1,994(2.4)$ & $46(2.1)$ & $83(2.2)$ & $1(0.8)$ \\
\hline Parous with PE and SGA & $250(0.3)$ & $61(2.9)^{*}$ & $15(0.4)$ & $1(0.8)$ \\
\hline Parous without PE but with SGA & $2,691(3.2)$ & $65(3.0)$ & $130(3.5)$ & $4(3.4)$ \\
\hline Cigarette smoker & $8,016(9.6)$ & $157(7.3)^{*}$ & $380(10.2)$ & $9(7.6)$ \\
\hline Family history of PE & $3,293(3.9)$ & $165(7.7)^{*}$ & $129(3.5)$ & $3(2.5)$ \\
\hline Assisted conception & $2,847(3.4)$ & $117(5.5)^{*}$ & $139(3.7)$ & $6(5.0)$ \\
\hline Chronic hypertension & $769(0.9)$ & $231(10.8)^{*}$ & $46(1.3)$ & $16(13.5)^{*}$ \\
\hline Pre-existing diabetes mellitus & $588(0.7)$ & $46(2.1)^{*}$ & $36(1.0)$ & $2(1.7)$ \\
\hline SLE/APS & $154(0.2)$ & $15(0.7)^{*}$ & $11(0.3)$ & 0 \\
\hline
\end{tabular}

Data are presented as median (interquartile range) or $\mathrm{n}(\%)$. Comparisons between outcome groups: $\chi^{2}$ test or Fisher's exact test for categorical variables and Mann-Whitney $U$ test or Student t test: ${ }^{*} \mathrm{p}<0.05$.

biochemical MoM values were simulated from the fitted multivariate Gaussian distribution for log-transformed MoM values. Thirdly, risks were obtained using the competing risk model from the simulated MoM values and the pregnancy characteristics for the $\mathrm{N}$ records. These three steps were applied to the pregnancies within the normal group with no restriction on the time of delivery. Fourthly, for a given FPR, risks from the normal group were used to define a risk cut-off. The proportion of PE risks was then used to obtain an estimate of the associated DR. The results presented are based on samples of $\mathrm{N}=10,000$ and the sampling error for a DR based on this sample size has a $95 \%$ error bound of $\pm 3 \%$.

The analyses were carried out using the R software [19], SPSS 20.0 (IBM SPSS Statistics for Windows, version 20.0; IBM Corp., Armonk, N.Y., USA) and Medcalc (Medcalc Software, Mariakerke, Belgium).

\section{Results}

\section{Characteristics of the Study Population}

The model for calculation of a priori risk based on maternal characteristics and history was derived from 2,140 cases of $\mathrm{PE}$ and 83,615 pregnancies unaffected by $\mathrm{GH}$ or SGA that were screened at 11-13 weeks' gestation [17]. The performance of screening by biochemical testing was derived from the study of pregnancies with measurements of serum PlGF and sFlt-1 (118 cases of PE and 3,734 unaffected pregnancies). The characteristics of the study populations are presented in table 1.

\section{Serum PlGF and sFlt-1 in Unaffected Pregnancies}

Multiple regression analysis demonstrated that for the prediction of the mean $\log _{10}$ PlGF, significant independent contributions were provided by maternal weight, racial origin (Afro-Caribbean and South Asian) and cigarette smoking $\left(\mathrm{R}^{2}=0.144\right.$; table 2$)$ but not gestational age at screening $(\mathrm{p}=0.055)$, maternal height $(\mathrm{p}=0.363)$ and age $(\mathrm{p}=0.069)$, obstetric history $(\mathrm{p}=$ $0.051)$, family history of PE ( $\mathrm{p}=0.652)$, method of conception $(p=0.585)$, chronic hypertension $(p=0.323)$, pre-existing diabetes mellitus $(\mathrm{p}=0.111)$ and SLE or APS $(p=0.305)$. 
Table 2. Fitted regression model for $\log _{10} \mathrm{PlGF}$ and sFlt- 1 at $30-33$ weeks

\begin{tabular}{lclccc}
\hline Coefficient & Estimate & Standard error & LCL & UCL & p value \\
\hline PlGF & & & & & \\
$\quad$ Intercept & 2.80061 & 0.02234 & 2.75683 & 2.84440 & 0 \\
$\quad$ (Gestation -210 days) & -0.0027448 & 0.0013035 & -0.0052997 & -0.0001899 & 0.0353 \\
(Weight $-69 \mathrm{~kg}$ ) & -0.0027980 & 0.0003080 & -0.0034018 & -0.0021943 & $<0.0001$ \\
Afro-Caribbean & 0.18181 & 0.01295 & 0.15642 & 0.20719 & $<0.0001$ \\
South Asian & 0.06395 & 0.02412 & 0.01668 & 0.11122 & 0.0080 \\
Smoking & 0.09036 & 0.01486 & 0.06125 & 0.11948 & $<0.0001$ \\
Parous & 0.03446 & 0.00929 & 0.01626 & 0.05267 & 0.0002 \\
sFlt-1 & & & & \\
Intercept & 3.22569 & 0.01495 & 3.19639 & 3.25499 & $<0.0001$ \\
(Gestation -210 days) & 0.0048664 & 0.0008806 & 0.0031404 & 0.0065924 & $<0.0001$ \\
(Weight -69 kg) & -0.0032132 & 0.0001929 & -0.0035913 & -0.0028351 & $<0.0001$ \\
(Maternal age -30 years) & 0.0017356 & 0.0005261 & 0.0007044 & 0.0027667 & 0.00098 \\
Afro-Caribbean & 0.10347 & 0.00805 & 0.08768 & 0.11925 & $<0.0001$ \\
Parous & -0.07530 & 0.00601 & -0.08709 & -0.06352 & $<0.0001$ \\
In vitro fertilization & 0.05544 & 0.01819 & 0.01980 & 0.09109 & 0.00231 \\
\hline LCL = Lower confidence limit; UCL $=$ upper confidence limit. & & & & \\
\hline
\end{tabular}

Table 3. Fitted regression model for marker $\log _{10}$ MoM values at 30-33 weeks of gestation at time of delivery for pregnancies with $\mathrm{PE}$

\begin{tabular}{lclccc}
\hline Marker & Estimate & SE & LCL & UCL & p value \\
\hline PlGF & & & & & \\
$\quad$ Intercept & -4.11583 & 0.45561 & -5.00882 & -3.22283 & $<0.0001$ \\
$\quad$ Slope & 0.095664 & 0.011845 & 0.072448 & 0.118879 & $<0.0001$ \\
sFlt-1 & & & & & \\
$\quad$ Intercept & 3.30026 & 0.28492 & 2.74181 & 3.85871 & $<0.0001$ \\
$\quad$ Slope & -0.080906 & 0.007408 & -0.095425 & -0.066388 & $<0.0001$ \\
\hline
\end{tabular}

Table 4. Standard deviations (SD) and correlations, with $95 \%$ confidence limits, for $\log _{10}$ MoM values for PlGF and sFlt-1

\begin{tabular}{lcc}
\hline & No PE & PE \\
\hline SD PlGF & $0.2965(0.2900$ to 0.3033$)$ & $0.2916(0.2587$ to 0.3342$)$ \\
SD sFlt-1 & $0.1969(0.1926$ to 0.2014$)$ & $0.2166(0.1921$ to 0.2482$)$ \\
Correlation & $-0.1113(-0.1424$ to -0.0800$)$ & $-0.3070(-0.4628$ to -0.1329$)$ \\
\hline
\end{tabular}

Multiple regression analysis demonstrated that for the prediction of the mean $\log _{10}$ sFlt-1 significant independent contributions were provided by gestational age at screening, maternal weight, Afro-Caribbean racial origin, IVF, prior history of PE and/or SGA, chronic hypertension and pre-existing diabetes mellitus $\left(\mathrm{R}^{2}=0.143\right.$; ta- ble 2) but not maternal height ( $\mathrm{p}=0.353)$, family history of PE $(\mathrm{p}=0.305)$, cigarette smoking $(\mathrm{p}=0.059)$ and SLE or APS $(p=0.297)$.

In each patient, we used these formulae to derive the expected $\log _{10}$ PlGF and $\log _{10}$ sFlt-1 at $30-33$ weeks, and then expressed the observed values as MoM of the ex- 
Fig. 1. Scatter diagram and regression line with 95\% confidence limits (interrupted lines) for the relationship between PlGF (a) and sFlt-1 (b) MoM at 30-33 weeks' gestational age and interval from screening to delivery in pregnancies with PE. The red horizontal line represents the 5 th percentile for PlGF and the 95th percentile for sFlt.

Fig. 2. Empirical DR with $95 \%$ confidence interval of PE requiring delivery within 4, 6 and 8 weeks of screening using maternal characteristics alone and maternal characteristics with biochemical markers, at FPR of $5 \%$. The red circles represent the modelled DRs for the same maternal characteristics.
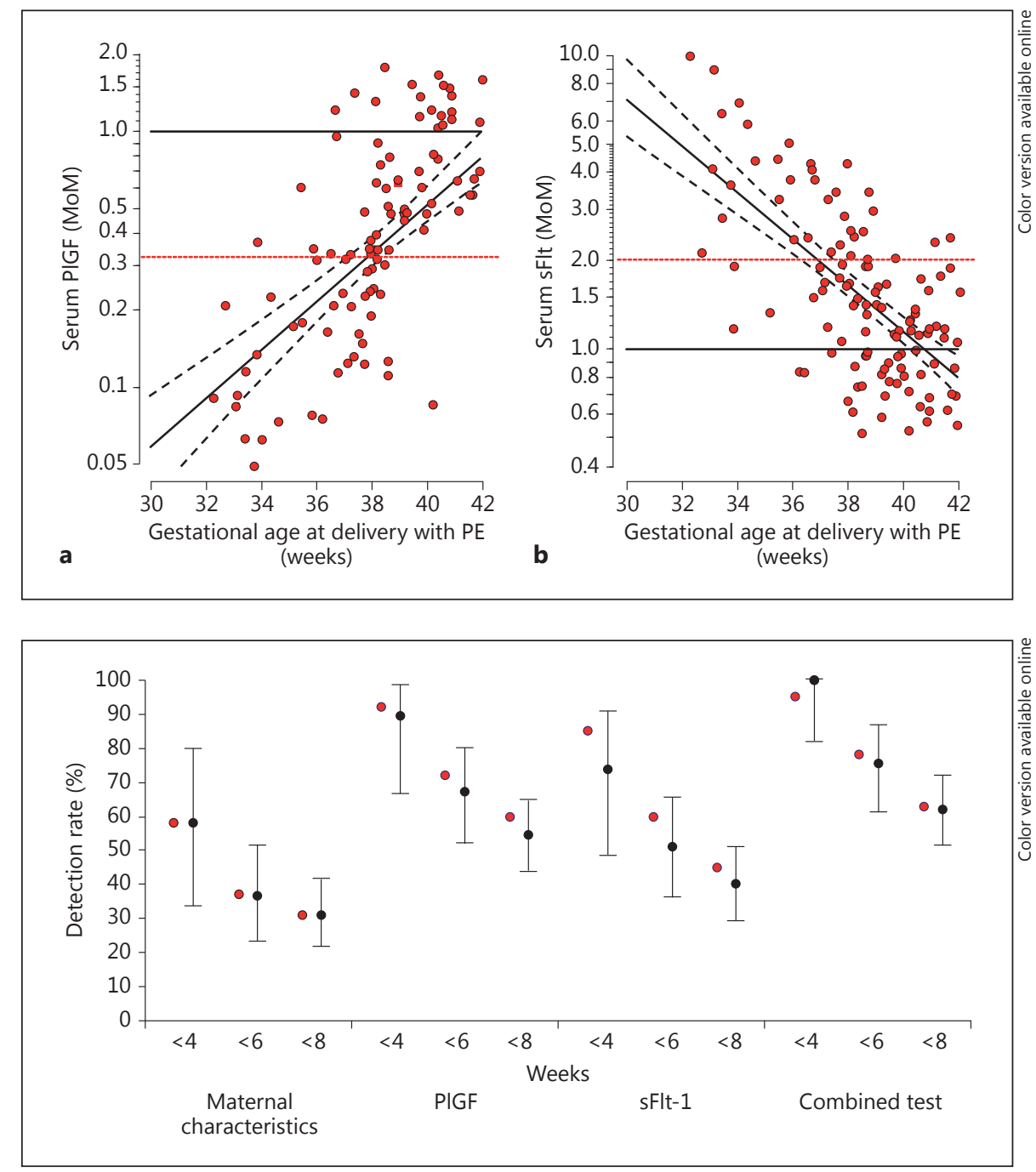

pected (table 3 ). Table 4 shows the standard deviations and correlations, with $95 \%$ confidence limits, for the respective $\log _{10}$ MoM values for PlGF and sFlt-1.

\section{Serum PlGF and sFlt-1 in Pregnancies with PE}

In the pregnancies with $\mathrm{PE}$, there was a significant direct association between $\log _{10}$ MoM PlGF with gestational age at delivery $(\mathrm{r}=0.604, \mathrm{p}<0.0001$; fig. 1$)$ and sampling to delivery interval $(\mathrm{r}=0.605, \mathrm{p}<0.0001$; fig. 1$)$. There was a significant inverse association between $\log _{10}$ MoM sFlt-1 with gestational age at delivery $(r=-0.622$, $\mathrm{p}<0.0001$; fig. 2) and sampling to delivery interval $(\mathrm{r}=$ -0.612 , $\mathrm{p}<0.0001$; fig. 2 ), between $\log _{10}$ sFlt-1-to-PlGF MoM ratio with gestational age at delivery $(r=-0.692$, $\mathrm{p}<0.0001)$ and sampling to delivery interval $(\mathrm{r}=-0.687$, $\mathrm{p}<0.0001)$ and between $\log _{10}$ MoM sFlt- 1 and $\log _{10} \mathrm{MoM}$ $\operatorname{PlGF}(\mathrm{r}=-0.564, \mathrm{p}<0.0001)$.

\section{Performance of Screening for PE}

The DR of all PE and PE requiring delivery within 4, 6 and 8 weeks of the visit, at fixed FPR of 5 and $10 \%$, in screening by maternal characteristics, PlGF and sFlt-1 and their combination are given in table 5 . The modelled and empirical performance was in good agreement with each other (fig. 2).

To provide estimates of performance in a large reference population, model-based results were obtained for the full sample of 83,615 normals and 2,140 cases of PE. Table 6 shows the performance of screening for PE requiring delivery within 4 weeks by a combination of ma- 
Table 5. Performance of screening for all PE and PE requiring delivery within 4, 6 and 8 weeks of screening using maternal characteristics alone and maternal characteristics with biochemical markers

\begin{tabular}{lllll}
\hline PE & Maternal characteristics & Serum PlGF & Serum sFlt- 1 & Combined testing \\
\hline $\begin{array}{l}\text { All PE }(\mathrm{n}=118) \\
\quad \text { FPR 5\% }\end{array}$ & $29(24.6 ; 17.1,33.4)$ & $54(45.8 ; 36.6,55.2)$ & $38(32.2 ; 23.9,41.4)$ & $63(53.4 ; 44,62.6)$ \\
$\quad$ FPR 10\% & $42(35.6 ; 27,44.9)$ & $74(62.7 ; 53.3,71.4)$ & $49(41.5 ; 32.5,51)$ & $75(63.6 ; 54.2,72.2)$ \\
PE <4 weeks $(\mathrm{n}=19)$ & & & \\
$\quad$ FPR 5\% & $11(57.9 ; 33.5,79.7)$ & $17(89.5 ; 66.9,98.7)$ & $14(73.7 ; 48.8,90.9)$ & $19(100 ; 82.4,100)$ \\
$\quad$ FPR 10\% & $12(63.2 ; 38.4,83.7)$ & $19(100 ; 82.4,100)$ & $18(94.7 ; 74,99.9)$ & $19(100 ; 82.4,100)$ \\
PE <6 weeks $(\mathrm{n}=49)$ & $18(36.7 ; 23.4,51.7)$ & $33(67.3 ; 52.5,80.1)$ & $25(51 ; 36.3,65.6)$ & $37(75.5 ; 61.1,86.7)$ \\
$\quad$ FPR 5\% & $22(44.9 ; 30.7,59.8)$ & $41(83.7 ; 70.3,92.7)$ & $35(71.4 ; 56.7,83.4)$ & $43(87.8 ; 75.2,95.4)$ \\
$\quad$ FPR 10\% & $28(31.1 ; 21.8,41.7)$ & $49(54.4 ; 43.6,65)$ & $36(40 ; 29.8,50.9)$ & $56(62.2 ; 51.4,72.2)$ \\
PE <8 weeks $(\mathrm{n}=90)$ & $36(40 ; 29.8,50.9)$ & $67(74.4 ; 64.2,83.1)$ & $50(55.6 ; 44.7,66)$ & $68(75.6 ; 65.4,84)$ \\
$\quad$ FPR 5\% & & & \\
$\quad$ FPR 10\% & & & \\
\hline
\end{tabular}

Number detected (estimated detection rate, \%; 95\% confidence interval) at FPR of 5 and 10\%. These results are based on the complete data subset of 118 cases of PE and 3,734 unaffected pregnancies.

Table 6. Modelled detection rates of PE requiring delivery within 4 weeks of screening and FPR, at risk cut-offs of 1:50 and 1:100 in screening by maternal factors, serum PlGF and sFlt-1 according to Caucasian and Afro-Caribbean racial origin and obstetric history

\begin{tabular}{|c|c|c|c|c|c|c|}
\hline \multirow[t]{2}{*}{ Study population } & \multicolumn{3}{|c|}{ Risk cut-off 1:50, \% } & \multicolumn{3}{|c|}{ Risk cut-off $1: 100, \%$} \\
\hline & FPR & DR & PPV & FPR & DR & PPV \\
\hline Total & 2.0 & 89.2 & 19.4 & 3.7 & 92.8 & 11.9 \\
\hline Caucasian all & 1.6 & 87.3 & 17.7 & 3.0 & 91.1 & 10.6 \\
\hline Caucasian nulliparous & 2.1 & 85.7 & 16.3 & 4.0 & 90.0 & 9.8 \\
\hline Caucasian parous without PE & 0.7 & 86.5 & 17.8 & 1.5 & 90.2 & 9.8 \\
\hline Caucasian parous with PE & 7.0 & 92.5 & 18.3 & 11.9 & 95.7 & 12.0 \\
\hline Afro-Caribbean all & 3.5 & 91.4 & 23.1 & 6.2 & 94.7 & 15.1 \\
\hline Afro-Caribbean nulliparous & 4.4 & 92.7 & 24.2 & 7.6 & 95.4 & 16.1 \\
\hline Afro-Caribbean parous without PE & 2.3 & 88.2 & 21.3 & 4.3 & 92.6 & 13.1 \\
\hline Afro-Caribbean parous with PE & 13.0 & 94.8 & 21.5 & 19.9 & 96.9 & 15.5 \\
\hline
\end{tabular}

These results relate to a population with the characteristics of the full sample of 2,140 cases of PE and 83,615 unaffected pregnancies. PPV $=$ Positive predictive value.

ternal factors, serum PlGF and sFlt-1 at risk cut-offs of 1:50 and 1:100 in the total population and in subgroups of women according to racial origin (Caucasian and AfroCaribbean) and obstetric history (nulliparous, parous with and without previous PE). At a risk cut-off of 1:50, the overall DR was $89.2 \%$ and FPR 2.0 with positive predictive value of 19.4 , and the respective values at risk cutoff of 1:100 were 92.8, 3.7 and 11.9\%. In women of AfroCaribbean racial origin, compared to Caucasians, and in nulliparous, compared to parous women, both the FPR and DR for PE were higher.

PlGF and sFlt-1 in Preeclampsia

\section{Discussion}

\section{Principal Findings of This Study}

This screening study for PE at 30-33 weeks' gestation examined prospectively a large population of pregnant women attending for routine care in a well-defined gestational age range which is widely used for the assessment of fetal growth and wellbeing. A survival time model was then developed that combines maternal characteristics and history, serum PlGF and sFlt- 1 to estimate the risk of developing PE requiring delivery within selected intervals 
from the time of screening. This approach assumes that if the pregnancy was to continue indefinitely, all women would develop PE and whether they do so or not before a specified gestational age depends on a competition between delivery before or after development of PE [15-17]. The effects of variables from maternal characteristics and history and biomarkers are to modify the mean of the distribution of gestational age at delivery with $\mathrm{PE}$, so that in pregnancies at low risk for PE the gestational age distribution is shifted to the right with the implication that in most pregnancies delivery will actually occur before the development of PE. In high-risk pregnancies, the distribution is shifted to the left and the smaller the mean gestational age the higher the risk for PE.

In normal singleton pregnancies at 30-33 weeks' gestation, serum PlGF decreases with gestational age and maternal weight and is higher in women of Afro-Caribbean and South Asian racial origin than in Caucasians, in parous than nulliparous women and in smokers than in non-smokers. Serum sFlt-1 increases with gestational age and maternal age, decreases with maternal weight, it is increased in women of Afro-Caribbean racial origin and in pregnancies conceived by IVF, and lower in parous than nulliparous women. Consequently, adjustments should be made for these maternal characteristics before valid comparisons can be carried out between normal and pathological pregnancies. Previous studies examining the value of serum PlGF or the sFlt-1 to PlGF ratio in the prediction of adverse outcome have not made adjustments for maternal characteristics [6-11].

In pregnancies complicated by $\mathrm{PE}$, compared to normal pregnancies, serum PlGF is decreased and sFlt- 1 is increased. The decrease in serum PlGF is likely to be the consequence of impaired trophoblastic invasion of the spiral arteries and their conversion from high impedance narrow vessels to wide non-muscular channels, which is thought to be the underlying cause of PE $[20,21]$. The hypoxic environment which results decreases PIGF expression in trophoblastic cells, which is reflected in the reduced circulating levels [22]. In contrast, hypoxia stimulates the upregulation of sFlt, which acts as an antagonist to PlGF, thereby exacerbating the angiogenic/anti-angiogenic imbalance [23, 24].

In pregnancies developing $\mathrm{PE}$, the deviation in $\mathrm{MoM}$ values of serum PlGF and sFlt-1 from normal are inversely related to the severity of the disease reflected in the gestational age at which delivery becomes necessary for maternal and or fetal indications. The findings of the study demonstrate that screening for PE at 30-33 weeks' gestation by a combination of maternal characteristics and se- rum PlGF and sFlt-1 can identify all cases developing PE and requiring delivery within the subsequent 4 weeks, at FPR of $5 \%$.

The FPR and DR of PE are influenced by the characteristics of the study population, and for a given risk cutoff they are both higher in women of Afro-Caribbean rather than Caucasian racial origin, and in nulliparous than in parous women with no previous PE. Consequently, comparison of the performance of screening using these algorithms between studies requires the appropriate adjustments for the characteristics of the population under investigation.

\section{Comparison with Findings of Previous Studies}

Previous studies investigating the performance of screening for PE have used specific cut-offs in serum PlGF and sFlt-1 concentrations or their ratio [6-11]. Such cut-offs have the advantage of simplicity in clinical practice. However, such approach does not take into account the a priori risk of the individual patient in the study population and ignores the effects of maternal characteristics on the measured serum concentrations and their interrelations in both normal and pathological pregnancies. Nevertheless, our findings of low serum PlGF and high sFlt-1 in pregnancies complicated by PE are consistent with those of previous studies investigating high-risk pregnancies which reported that measurement of serum PlGF or the sFlt- 1 to PlGF ratio are highly accurate in identifying the subgroup that will develop severe $\mathrm{PE}$ requiring delivery within the subsequent few weeks [6-11]. Our results are also consistent with those of a previous screening study which examined 1,269 singleton pregnancies at 30-34 weeks' gestation and reported that the ratio could identify $58 \%$ of their 40 cases of PE, at FPR of $15 \%$ [25].

In a previous screening study at 30-33 weeks' gestation, we used a survival time model for the time of delivery for PE by combination of maternal characteristics and history with mean arterial pressure and uterine artery pulsatility index [17]. This approach detected 90, 65, and $53 \%$ of PE with delivery within 4,6 and 8 weeks of the visit, at fixed FPR of $5 \%$.

\section{Implications for Clinical Practice and Future Research}

Extensive research in the last decade has led to the proposal of a two-stage strategy for identification of pregnancies at high-risk of developing PE, the first stage at 11-13 weeks' gestation and the second at 30-33 weeks [26]. The objective of first-trimester screening is the identification of pregnancies at high risk of preterm PE 
and through pharmacological intervention in this highrisk group the reduction in the prevalence of the disease $[16,27-29]$.

The objective of screening for PE at $30-33$ weeks is to effectively predict $\mathrm{PE}$ developing within the subsequent few weeks, because close monitoring of such pregnancies for earlier diagnosis of the clinical signs of the disease could potentially improve perinatal outcome through such interventions as the administration of antihypertensive medication and early delivery [30]. The potential value of novel treatments, including the administration of statins and VEGF or extracorporeal removal of sFlt-1, is currently under investigation [31-33]. Future studies will examine whether the performance of screening for late $\mathrm{PE}$ can be improved by combining biophysical and biochemical tests at 30-33 weeks or by repeat testing at around 37 weeks' gestation.

\section{Acknowledgement}

This study was supported by a grant from the Fetal Medicine Foundation (Charity No. 1037116). The machine and reagents for the assays were provided by Roche Diagnostics Limited.

\section{References}

1 World Health Organization: Make Every Mother and Child Count. World Health Report, 2005. Geneva, World Health Organization, 2005.

2 Confidential Enquiry into Maternal and Child Health (CEMACH) Perinatal Mortality 2006: England, Wales and Northern Ireland. London, CEMACH, 2008.

3 Duley L: The global impact of pre-eclampsia and eclampsia. Semin Perinatol 2009;33:130 137.

4 Bdolah Y, Sukhatme VP, Karumanchi SA: Angiogenic imbalance in the pathophysiology of preeclampsia: newer insights. Semin Nephrol 2004;24:548-556.

5 Kleinrouweler C, Wiegerinck M, Ris-Stalpers C, Bossuyt P, van der Post J, von Dadelszen P, Mol B, Pajkrt E, for the EBM CONNECT Collaboration: Accuracy of circulating placental growth factor, vascular endothelial growth factor, soluble fms-like tyrosine kinase 1 and soluble endoglin in the prediction of pre-eclampsia: a systematic review and meta-analysis. BJOG 2012;119:778-787.

-6 Verlohren S, Herraiz I, Lapaire O, Schlembach D, Moertl M, Zeisler H, Calda P, Holzgreve W, Galindo A, Engels T, Denk B, Stepan H: The sFlt-1/PlGF ratio in different types of hypertensive pregnancy disorders and its prognostic potential in preeclamptic patients. Am J Obstet Gynecol 2012;206:58 e1-e8.

7 Rana S, Powe CE, Salahuddin S, Verlohren S, Perschel FH, Levine RJ, Lim KH, Wenger JB, Thadhani R, Karumanchi SA: Angiogenic factors and the risk of adverse outcomes in women with suspected preeclampsia. Circulation 2012;125:911-919.

$>8$ Chaiworapongsa T, Romero R, Savasan ZA, Kusanovic JP, Ogge G, Soto E, Dong Z, Tarca A, Gaurav B, Hassan SS: Maternal plasma concentrations of angiogenic/anti-angiogenic factors are of prognostic value in patients presenting to the obstetrical triage area with the suspicion of preeclampsia. J Matern Fetal Neonatal Med 2011;24:1187-207.

-9 Sibiude J, Guibourdenche J, Dionne MD, Le Ray C, Anselem O, Serreau R, Goffinet F, Tsatsaris V: Placental growth factor for the prediction of adverse outcomes in patients with suspected preeclampsia or intrauterine growth restriction. PLoS One 2012;7:e50208.

10 Chappell LC, Duckworth S, Seed PT, Griffin M, Myers J, Mackillop L, Simpson N, Waugh J, Anumba D, Kenny LC, Redman CW, Shennan AH: Diagnostic accuracy of placental growth factor in women with suspected preeclampsia: a prospective multicenter study. Circulation 2013;128:2121-2131.

11 Ohkuchi A, Hirashima C, Takahashi K, Suzuki H, Matsubara S, Suzuki M: Onset threshold of the plasma levels of soluble fms-like tyrosine kinase $1 /$ placental growth factor ratio for predicting the imminent onset of preeclampsia within 4 weeks after blood sampling at 19-31 weeks of gestation. Hypertens Res DOI: 10.1038/hr.2013.95.

12 Robinson HP, Fleming JE: A critical evaluation of sonar crown rump length measurements. Br J Obstet Gynaecol 1975;82:702-710.

13 Brown MA, Lindheimer MD, de Swiet M, Van Assche A, Moutquin JM: The classification and diagnosis of the hypertensive disorders of pregnancy: statement from the International Society for the Study of Hypertension in Pregnancy (ISSHP). Hypertens Pregnancy 2001; 20:IX-XIV.

14 Poon LCY, Volpe N, Muto B, Syngelaki A, Nicolaides KH: Birthweight with gestation and maternal characteristics in live births and stillbirths. Fetal Diagn Ther DOI: $10.1159 / 000338655$.

15 Wright D, Akolekar R, Syngelaki A, Poon LC, Nicolaides $\mathrm{KH}$ : A competing risks model in early screening for preeclampsia. Fetal Diagn Ther 2012;32:171-178.

16 Akolekar R, Syngelaki A, Poon L, Wright D, Nicolaides KH: Competing risks model in early screening for preeclampsia by biophysical and biochemical markers. Fetal Diagn Ther 2013;33:8-15.

17 Tayyar A, Garcia-Tizon Larroca S, Poon LC, Wright D, Nicolaides KH: Competing risks model in screening for preeclampsia by mean arterial pressure and uterine artery pulsatility index at 30-33 weeks' gestation. Fetal Diagn Ther 2013;33:8-15.

18 Kalbfleisch JD, Prentice RL: The Statistical Analysis of Failure Time Data, ed 2. New York, Wiley, 2002.

19 R Core Team: R: a language and environment for statistical computing. Vienna, R Foundation for Statistical Computing, 2013. http:// www.R-project.org/.

20 Khong TY, De Wolf F, Robertson WB, Brosens I: Inadequate maternal vascular response to placentation in pregnancies complicated by pre-eclampsia and by small-for-gestational age infants. BJOG 1986;93:1049-1059.

21 Pijnenborg R, Anthony J, Davey DA, Rees A Tiltman A, Vercruysse L, van Assche A: Placental bed spiral arteries in the hypertensive disorders of pregnancy. BJOG 1991;98:648655

22 Torry DS, Mukherjea D, Arroyo J, Torry RJ Expression and function of placenta growth factor: implications for abnormal placentation. J Soc Gynecol Investig 2003;10:178-188.

23 Munaut C, Lorquet S, Pequeux C, Blacher S, Berndt S, Frankenne F, Foidart JM: Hypoxia is responsible for soluble vascular endothelial growth factor receptor-1 (VEGFR-1) but not for soluble endoglin induction in villous trophoblast. Hum Reprod 2008;23:1407-1415.

24 Maynard SE, Min JY, Merchan J, Lim KH, Li J, Mondal S, Libermann TA, Morgan JP, Sellke FW, Stillman IE, Epstein FH, Sukhatme VP, Karumanchi SA: Excess placental soluble fms-like tyrosine kinase 1 (sFlt1) may contribute to endothelial dysfunction, hypertension, and proteinuria in preeclampsia. J Clin Invest 2003;111:649-658. 
25 Chaiworapongsa T, Romero R, Korzeniewski SJ, Kusanovic JP, Soto E, Lam J, Dong Z, Than NG, Yeo L, Hernandez-Andrade E, CondeAgudelo A, Hassan SS: Maternal plasma concentrations of angiogenic/antiangiogenic factors in the third trimester of pregnancy to identify the patient at risk for stillbirth at or near term and severe late preeclampsia. Am J Obstet Gynecol 2013;208:287.e1-287.e15.

26 Nicolaides KH: Turning the pyramid of prenatal care. Fetal Diagn Ther 2011;29:183-196.

-27 Poon LC, Kametas NA, Maiz N, Akolekar R, Nicolaides KH: First-trimester prediction of hypertensive disorders in pregnancy. Hypertension 2009;53:812-818.

-28 Bujold E, Roberge S, Lacasse Y, Bureau M, Audibert F, Marcoux S, Forest JC, Giguère Y: Prevention of preeclampsia and intrauterine growth restriction with aspirin started in early pregnancy: a meta-analysis. Obstet Gynecol 2010;116:402-414.
29 Roberge S, Villa P, Nicolaides K, Giguère Y, Vainio M, Bakthi A, Ebrashy A, Bujold E: Early administration of low dose aspirin for the prevention of preterm and term pre-eclampsia: a systematic review and meta-analysis. Fetal Diagn Ther 2012;31:141-146.

30 Koopmans CM, Bijlenga D, Groen H, Vijgen SM, Aarnoudse JG, Bekedam DJ, van den Berg PP, de Boer K, Burggraaff JM, Bloemenkamp KW, Drogtrop AP, Franx A, de Groot CJ, Huisjes AJ, Kwee A, van Loon AJ, Lub A, Papatsonis DN, van der Post JA, Roumen FJ, Scheepers HC, Willekes C, Mol BW, van Pampus MG; HYPITAT study group: Induction of labour versus expectant monitoring for gestational hypertension or mild pre-eclampsia after 36 weeks' gestation (HYPITAT): a multicentre, open-label randomised controlled trial. Lancet 2009;374:979-988.
Kumasawa K, Ikawa M, Kidoya H, Hasuwa H, Saito-Fujita T, Morioka Y, Takakura N, Kimura T, Okabe M: Pravastatin induces placental growth factor (PGF) and ameliorates preeclampsia in a mouse model. Proc Natl Acad Sci USA 2011;108:1451-1455.

32 Li Z, Zhang Y, Ying Ma J, Kapoun AM, Shao Q, Kerr I, Lam A, O’Young G, Sannajust F, Stathis P, Schreiner G, Karumanchi SA, Protter AA, Pollitt NS: Recombinant vascular endothelial growth factor 121 attenuates hypertension and improves kidney damage in a rat model of preeclampsia. Hypertension 2007;50:686-692.

33 Thadhani R, Kisner T, Hagmann H, Bossung V, Noack S, Schaarschmidt W, Jank A, Kribs A, Cornely OA, Kreyssig C, Hemphill L, Rigby AC, Khedkar S, Lindner TH, Mallmann P, Stepan H, Karumanchi SA, Benzing T: Pilot study of extracorporeal removal of soluble fms-like tyrosine kinase 1 in preeclampsia. Circulation 2011;124:940-950. 